\title{
A New Algorithm for Shoreline Extraction from Satellite Imagery with Non-Separable Wavelet and Level Set Method
}

\author{
Shujian Yu, Yi Mou, Duanquan Xu, Xinge You, Long Zhou, and Wu Zeng
}

\begin{abstract}
An effective and precise method for shoreline detection from satellite imagery is presented. The algorithm is based on two main steps: (1) the detection of singularities in a single image using non-separable wavelet and (2)amendment procedure using distance regularized level set evolution scheme. Firstly, by selecting appropriate parameters, the non-separable wavelet filter banks which can provide information of different orientations are used to capture the singularities of the selected single satellite image; Secondly, obtaining the modulus image by utilizing sub-images decomposed from the non-separable wavelet filter banks; Thirdly, extracting the shoreline iteratively with the use of distance regularized level set evolution scheme. Experiments are conducted and results show that the proposed algorithm is applicable to satellite imagery, and the shoreline is robust to noises as well as blurring.
\end{abstract}

Index Terms-Shoreline detection, non-separable wavelet, Satellite imagery, edge detection, level set method, distance regularized level set evolution.

\section{INTRODUCTION}

The detection and extraction of shoreline is of great importance in several applications such as cartography and the environmental management of the entire coastal zone. Knowledge of shoreline can be utilized for measuring and characterizing land and water resources and the perimeter of shoreline. Information about shoreline position, orientation and geometric shape is also essential for autonomous navigation, geographical exploration, coastal erosion monitoring and modeling, and coastal resource inventory and management [1]

Conventionally, the shoreline is defined as the physical interface between land and water [2]. According to the definition, there are two factors which can determine the position of the shoreline: (1) short-term variations in sea level that caused by astronomical and meteorological factors [3]; and (2)alterations in the shapes and volumes of sediments along the profile of shore.

The above two types of factors are essential for the management of coastal areas. The first type of factor reveals a definite tendency and is more important as it enables

Manuscript received September 13, 2012; revised December 27, 2012. This work was supported partially by the NSF under grant 60973154 and 61075015, the Program of International Science and Technology Cooperation (No.2011DFA12180), Ph.D. Programs Foundation of Ministry of Education of China (No.20110142110060), the National Key Technology R\&D Program (No.2012BAK02B06), Natural Science Foundation of Hubei Province(No.2010CDA006)

Duanquan $\mathrm{Xu}$ is with Electronics and Information Engineering, Huazhong University of Science and Technology, Wuhan 430074, China (e-mail: xuduanquan@126.com). predictions to be made about whether or not the shore is subject to significant changes. And the second type of factor discloses the magnitude of the variability over a long time and therefore enables a feasible coastal management plan to define and establish coastal protection area.

For these reasons, to detect and extract the shoreline from satellite imagery has attracted many attentions [4], [5]. Boak and Tunner[6] described up to 44 different indicators to locate the shoreline used by different authors from the 1950s until today. There are generally two types of approaches: either a feature that is visibly discernible be utilized in coastal imagery or the interSection of a tidal datum with the coastal profile is detected as the shoreline.

The disadvantages and challenges of the existing methods are: (1)most of them are manual, which means the research people must specify some key points or other prior knowledge for the extraction methods[7]-[9]. Due to the subjectivity and substantial effort involved in manual delineation, an automatic or semi-automatic method has been long desired; (2)some of the methods only applicable for one type of satellite imagery and disregard other images acquired from other satellites, therefore, an adaptive methodology which can be utilized for most of the satellite imagery, such as Landsat imagery, SPOT imagery and CBERS imagery, is also desired; (3)the images acquired from different some remote platforms are often blurred due to immature imaging technology, and a robust methodology to noises and blurring is essential for researchers; and (4)shoreline is interface between land and water, and, in other words, shoreline represents singularities of a satellite imagery. Unfortunately, these methods do not take the singularities of the shorelines into consideration.

In summary, the current method to detect and extract shoreline should mainly consider the criteria of objectivity, automaticity, and robustness. From image processing point of view, the singularities represent the high frequency components of image[10]-[13]. Employing singularities to locate shoreline should be given more high frequency components. Wavelet transform is a time-frequency analysis tools which is wildly used in signal and image processing fields. It has the property of multi-resolution with which we can analyze signal at different scales. Therefore, in the application of singularity detection, we can employ comprehensive information of the signal at different scales to judge if the points belong to an edge. This is the superiority of wavelet transform compared with other edge detection method. In addition, traditional wavelet is separable which means when it is utilized in image processing, we can divide the whole procedure into two steps: (1) performing wavelet 
transform along vertical direction and (2) performing wavelet transform along horizontal direction. Most importantly, the two steps are exchangeable, which means this kind of procedure cannot detect much singular information as well as the direction information. However, non-separable wavelet transform cannot be divided into such two steps due to its special decimation such as: quincunx decimation. Compared with separable wavelet, non-separable wavelet is capable of extracting more high frequency components especially the direction information from original image. So we proposed a shoreline extraction algorithm which combines non-separable wavelet transform with an amendment processing which is from level set method [14], and the results are shown in Fig 1.

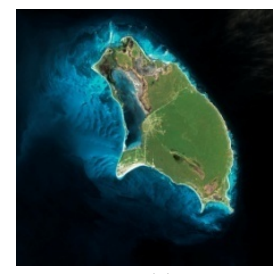

(a)

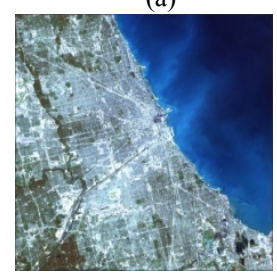

(c)

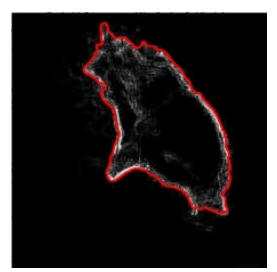

(b)

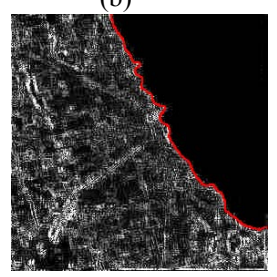

(d)
Fig. 1. shoreline extracted by our algorithm: (a) and (c) are original image from SPOT satellite and Landsat satellite, respectively; (b) and (d) are the extracted shorelines

The remainder of this paper is organized as follows: In Section II, the sources of the original images for our research are demonstrated and in Section III, non-separable wavelet construction is briefly introduced. Then, the amendment processing from level set method by using distance regularized level set evolution is introduced in Section IV. Experiment results and results analysis are presented in Section V. And finally, in Section VI, the conclusions are drawn.

\section{IMAGE DATA SOURCE}

The data sources used for our research are downloaded from the homepages of seven different satellites, namely Landsat-7, SPOT-5, CBERS-2, WorldView-2, Aster, Quick-Bird and IKONOS. The seven satellites are representative for remote sensing, and some characters of them are shown in Table I. And the pictures can be download from:

http://landsat.gsfc.nasa.gov/,http://www.astrium-geo.com/cn /,http://www.satimagingcorp.com/

To verify the validity of our algorithm to all, at least the most of, different satellites imagery, we randomly selected 80 images from the downloaded images of different satellites and established an image set for our future research (images from the same satellite are collected into the same category). However, in this paper, we will only primarily utilize three images from our image set to demonstrate how good our methodology is. And Table II identifies the three images.

TABLE I: SATELLITE IDENTITIES

\begin{tabular}{llc}
\hline \hline Satellite & Orbit & Resolution \\
\hline Landsat-7 & $705 \mathrm{~km}$ altitude, Sun synchronous & 15 to 90 meters \\
SPOT-5 & $822 \mathrm{~km}$ altitude, Sun synchronous & \\
WorldView-2 & $770 \mathrm{~km}$ altitude, Sun synchronous & $\begin{array}{c}\text { Please browse } \\
\text { website for detail } \\
\text { information. }\end{array}$ \\
Quick-Bird & $450 \mathrm{~km}$ altitude, Sun synchronous & \\
IKONOS & $681 \mathrm{~km}$ altitude, Sun synchronous & \\
CBERS-2 & $778 \mathrm{~km}$ altitude, Sun synchronous & $20 \mathrm{~m}-260 \mathrm{~m}$ \\
Aster & $705 \mathrm{~km}$ altitude, Sun synchronous & 15 to 90 meters \\
\hline \hline
\end{tabular}

TABLE II: IMAGE IDENTITIES

\begin{tabular}{lll}
\multicolumn{2}{c}{ TABLE II: IMAGE IDENTITIES } \\
\hline \hline Image & Satellite & Data of Requisition \\
\hline Cyprus & Landsat-7 & Fall of 2002 \\
Puducherry of India & SPOT-5 & March $26^{\text {th }}$ of 2007 \\
South America & CBERS-2 & Not disclose \\
\hline \hline
\end{tabular}

\section{Methodology}

The overall flow is shown as Fig 2: differentiated form the traditional wavelet transforms, the non-separable wavelet transform is capable of extracting more high frequency components from images with different orientations via properly selecting parameters. Using non-separable wavelet transform to a single image, we can get the coefficients which corresponding to different filters. Based on the coefficients, we can then calculate the modulus and get the approximate shorelines of the experimental image.

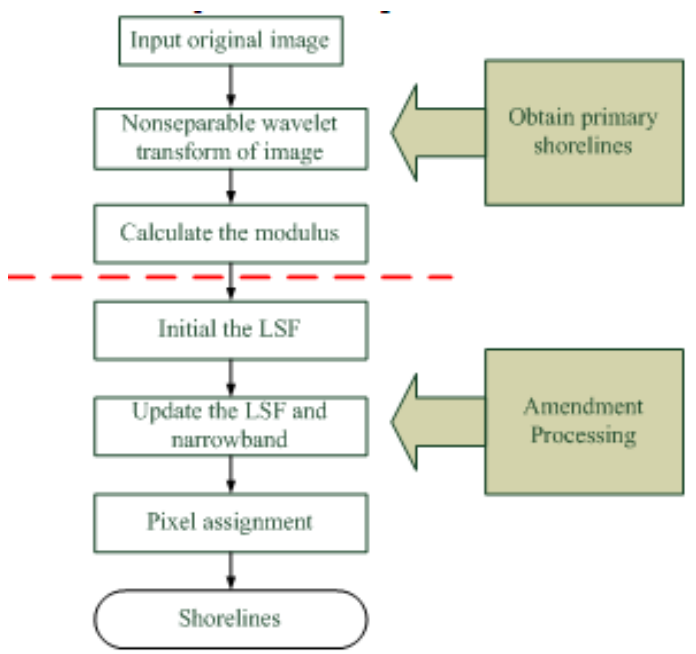

Fig. 2. Basic flow of proposed method

The terminology of LSF is from the level set method [15]-[17], which is widely used in image processing and computer vision. According to level set method, the basic idea is to represent a contour as the zero level set of a higher dimensional function, which is called level set function (LSF), and in our algorithm, we will initialize a proper LSF firstly in the second part. After then, we will formulate the motion of the contour as the evolution of the level set function according to the level set method. In addition in this procedure, we will bring in a new type of level set evolution called distance regularized level set evolution (DRLSE). The distance regularization effect eliminates the need for re-initialization and thereby avoids its induced numerical 
errors. Finally, we will assign values to new pixels on the narrowband according to the assignment algorithm. Consequently, through the above algorithm we will get a clear shoreline from each experimental image.

\section{A. Construction of Non-Separable Wavelet}

In our prior works [18]-[19], a non-separable wavelets is constructed by using centrally symmetric matrix $S_{(\alpha, \beta)}$,where $\alpha$ and $\beta$ are parameters. From the centrally symmetric matrix, we may derive a class of the non-separable wavelet below:

The low pass filter $m_{0}\left(z_{1}, z_{2}\right)$ and high pass filter $m_{j}\left(z_{1}, z_{2}\right)(j=1,2,3)$ are defined as follow:

$m_{j}\left(z_{1}, z_{2}\right)=\frac{1}{4}\left(1, z_{1}, z_{2}, z_{1} z_{2}\right)\left(\prod_{t=1}^{N} S_{\left(\alpha_{t}, \beta_{t}\right)} D\left(z_{1}^{2}, z_{2}^{2}\right) S_{\left(\alpha_{t}, \beta_{t}\right)}^{T}\right) V_{j}$ where $\left(z_{1}, z_{2}\right) \in \partial D \times \partial D, j=0,1,2,3$.

$D=z:|z| \leq 1, \partial D=z:|z|=1, V_{0}=(1,1,1,1)^{T}, V_{1}=(1,-1,1,-1)^{T}$

$V_{2}=(1,1,-1,-1)^{T}, V_{3}=(1,-1,-1,1)^{T}$

$S_{\left(\alpha_{t}, \beta_{t}\right)}$ is centrally symmetric orthogonal matrix, and $D\left(\mathrm{z}_{1}, \mathrm{z}_{2}\right)$ is the matrix of trigonometric polynomial below:

$$
D\left(z_{1}, z_{2}\right)=\left[\begin{array}{cccc}
1 & 0 & 0 & 0 \\
0 & z_{1} & 0 & 0 \\
0 & 0 & z_{2} & 0 \\
0 & 0 & 0 & z_{1} z_{2}
\end{array}\right],\left(z_{1}, z_{2}\right) \in \partial D \times \partial D
$$

and the matrix:

$$
S_{(\alpha, \beta)}=\frac{1}{2}\left(\begin{array}{cccc}
z_{1}+z_{3} & -z_{2}+z_{4} & -z_{2}-z_{4} & z_{1}-z_{3} \\
z_{2}-z_{4} & z_{1}+z_{3} & z_{1}-z_{3} & z_{2}+z_{4} \\
z_{2}+z_{4} & z_{1}-z_{3} & z_{1}+z_{3} & z_{2}-z_{4} \\
z_{1}-z_{3} & -z_{2}-z_{4} & -z_{2}+z_{4} & z_{1}+z_{3}
\end{array}\right)
$$

where $z_{1}=\cos \alpha, z_{2}=\sin \alpha \quad, z_{3}=\cos \beta, z_{4}=\sin \beta$, according to (1),(2) and (3) the size of the filters relay is dependent on the number of $N$. Explicitly, the relationship is

$$
\text { filtersize }=2(N+1) \times 2(N+1)
$$

By selecting different parameters, we could get different wavelet filters. For example, if $N=3,\left(\alpha_{1}=\pi / 4, \beta_{1}\right.$ $=\pi / 16),\left(\alpha_{2}=\pi / 3, \beta_{2}=\pi / 256\right),\left(\alpha_{3}=\pi / 5\right.$,

$\left.\beta_{3}=4 \pi / 7\right)$, filters $m_{0}, m_{1}, m_{2}, m_{3}$ are:

$m_{0}=\left(\begin{array}{cccccccc}0.2019 & 0.0612 & 0.1392 & 0.0922 & 0.1090 & -0.3855 & -0.0097 & 0.0320 \\ 0.1079 & -0.0327 & 0.0744 & -0.0493 & 0.0583 & 0.2061 & -0.0052 & -0.0171 \\ -0.0365 & -0.0111 & 0.3057 & 0.0837 & -0.0806 & 0.1332 & -0.0492 & 0.1623 \\ -0.0094 & 0.0029 & 0.1704 & -0.0493 & -0.0376 & -0.0519 & -0.0268 & -0.0883 \\ -0.0883 & -0.0268 & -0.0519 & -0.0376 & -0.0493 & 0.1704 & 0.0029 & -0.0094 \\ 0.1623 & -0.0492 & 0.1332 & -0.0806 & 0.0837 & 0.3057 & -0.0111 & -0.0365 \\ -0.0171 & -0.0052 & 0.2061 & 0.0583 & -0.0493 & 0.0744 & -0.0327 & 0.1079 \\ 0.0320 & -0.0097 & -0.3855 & 0.1090 & 0.0922 & 0.1392 & 0.0612 & 0.2019\end{array}\right)$

$m_{1}=\left(\begin{array}{cccccccc}0.1088 & 0.0330 & 0.3819 & 0.1428 & 0.0023 & -0.1488 & -0.0525 & 0.1731 \\ 0.0581 & -0.0176 & 0.2041 & -0.0763 & 0.0012 & 0.0795 & -0.0281 & -0.0925 \\ 0.1349 & 0.0409 & -0.1408 & -0.0093 & 0.1158 & -0.3023 & 0.0295 & -0.0974 \\ 0.0775 & -0.0235 & -0.0562 & -0.0021 & 0.0620 & 0.1690 & 0.0132 & 0.0434 \\ -0.0434 & -0.0132 & -0.1690 & -0.0620 & 0.0021 & 0.0562 & 0.0235 & -0.0775 \\ 0.0974 & -0.0295 & 0.3023 & -0.1158 & 0.0093 & 0.1408 & -0.0409 & -0.1349 \\ 0.0925 & 0.0281 & -0.0795 & -0.0012 & 0.0763 & -0.2041 & 0.0176 & -0.0581 \\ -0.1731 & 0.0525 & 0.1488 & -0.0023 & -0.1428 & -0.3819 & -0.0330 & -0.1088\end{array}\right)$
$m_{2}=\left(\begin{array}{cccccccc}-0.1731 & -0.0525 & 0.1488 & 0.0023 & -0.1428 & 0.3819 & -0.0330 & 0.1088 \\ -0.0925 & 0.0281 & 0.0795 & -0.0012 & -0.0763 & -0.2041 & -0.0176 & -0.0581 \\ 0.0974 & 0.0295 & 0.3023 & 0.1158 & 0.0093 & -0.1408 & -0.0409 & 0.1349 \\ 0.0434 & -0.0132 & 0.1690 & -0.0620 & -0.0021 & 0.0562 & -0.0235 & -0.0775 \\ 0.0775 & 0.0235 & -0.0562 & 0.0021 & 0.0620 & -0.1690 & 0.0132 & -0.0434 \\ -0.1349 & 0.0409 & 0.1408 & -0.0093 & -0.1158 & -0.3023 & -0.0295 & -0.0974 \\ 0.0581 & 0.0176 & 0.2041 & 0.0763 & 0.0012 & -0.0795 & -0.0281 & 0.0925 \\ -0.1088 & 0.0330 & -0.3819 & 0.1428 & -0.0023 & -0.1488 & 0.0525 & 0.1731\end{array}\right)$
$m_{3}=\left(\begin{array}{ccccccccc}0.0320 & 0.0097 & -0.3855 & -0.1090 & 0.0922 & -0.1392 & 0.0612 & -0.2019 \\ 0.0171 & -0.0052 & -0.2061 & 0.0583 & 0.0493 & 0.0744 & 0.0327 & 0.1079 \\ 0.1623 & 0.0492 & 0.1332 & 0.0806 & 0.0837 & -0.3057 & -0.0111 & 0.0365 \\ 0.0883 & -0.0268 & 0.0519 & -0.0376 & 0.0493 & 0.1704 & -0.0029 & -0.0094 \\ -0.0094 & -0.0029 & 0.1704 & 0.0493 & -0.0376 & 0.0519 & -0.0268 & 0.0883 \\ 0.0365 & -0.0111 & -0.3057 & 0.0837 & 0.0806 & 0.1332 & 0.0492 & 0.1623 \\ 0.1079 & 0.0327 & 0.0744 & 0.0493 & 0.0583 & -0.2061 & -0.0052 & 0.0171 \\ -0.2019 & 0.0612 & -0.1392 & 0.0922 & -0.1090 & -0.3855 & 0.0097 & 0.0320\end{array}\right)$

The parameters of filters we employed [20], are listed in Table III, and the sub-images processed by filters are shown in Fig. 3.

TABLE III: PARAMETERS OF EMPLOYED FILTERS

\begin{tabular}{ccc}
\hline \hline Orientation & Parameters $N=3(k=1,2,3)$ & Filter \\
\hline$\rho=\pi / 4$ & $\alpha_{k}=31 \pi / 50, \beta_{k}=9 \pi / 50$ & $m_{2}$ \\
$\rho=\pi / 2$ & $\alpha_{k}=33 \pi / 50, \beta_{k}=3 \pi / 50$ & $m_{2}$ \\
$\rho=3 \pi / 4$ & $\alpha_{k}=21 \pi / 50, \beta_{k}=2 \pi / 50$ & $m_{1}$ \\
$\rho=\pi$ & $\alpha_{k}=12 \pi / 25, \beta_{k}=13 \pi / 25$ & $m_{1}$ \\
\hline \hline
\end{tabular}

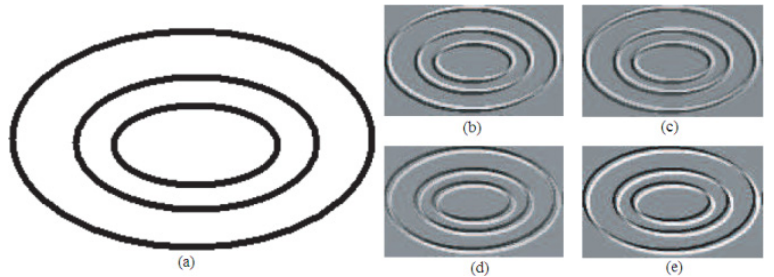

Fig. 3. Sub-images of four non-separable wavelet filters: (a)original image;(b) $\rho=\pi / 4$; (c) $\rho=\pi / 2$;(d) $\rho=3 \pi / 4$ and (e) $\rho=\pi$

\section{B. Amendment Processing}

Amendment processing procedure which is from the level set method is based on the distance regularized level set evolution[14].the algorithm is as follows:

Initialization: Initialize level set function(LSF) $\psi$ as $\psi_{i n}$, and narrowband $B_{r}^{0}=\bigcup_{i, j \in Z} 0 N_{i, j}^{r}$ where $Z^{0}$ is the set of the zero crossing points of $\psi_{i n}$;

Update the LSF: $\psi_{i, j}^{k+1}=\psi_{i, j}^{k}+\tau L\left(\psi_{i, j}^{k}\right)$ on the narrowband where $k=0,1,2, \cdots$;

Update the narrowband: Define set $Z^{k+1}$ as a set of zero crossing pixels of $\psi_{i, j}^{k+1}$ on $B_{r}^{k}$ update criteria $B_{r}^{k+1}=\bigcup_{i, j \in Z^{k+1}} N_{i, j}^{r}$ 
Assign value to new pixel on the narrowband: Every pixel $(i, j)$ in $B_{r}^{k+1}$ but not in $B_{r}^{k}$,set $\psi_{i, j}^{k+1}$ to $h$ if $\psi_{i, j}^{k}>0$,or else set $\psi_{i, j}^{k+1}$ to $-h$,where $h$ is a constant, which can be set to $r+1$ as a default value;

Determine the topping condition: If either the zero crossing points stop varying for consecutive iterations or exceeds a prescribed maximum number of iterations, then stop the iteration, otherwise, go to Step 2. And the codes can be download from: http://www.engr.uconn.edu/ cmli/.

\section{EXPERIMENTS}

We have conducted two types of experiments in our research: (1) detecting and extracting shoreline directly from different satellite imagery, and (2) testifying the robustness of our algorithm to noises and blurring. The results are shown in Fig.4-Fig.9.



(a)

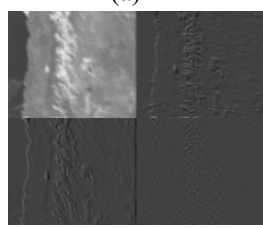

(d)



(b)



(e)

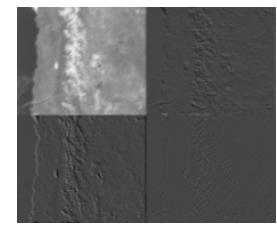

(c)



(f)
Fig. 4. Shoreline extracted directly from the CBERS-2 satellite image. (a) is original image; (b)-(e) are the images processed via non-separable wavelet filters with different parameters; (f) is the extracted shoreline which is plotted by the red curve.

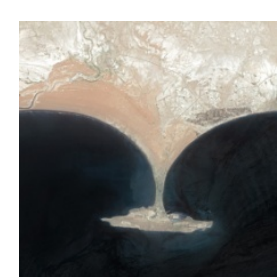

(a)

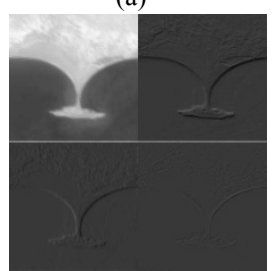

(d)

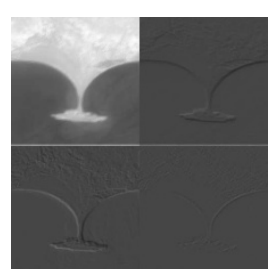

(b)

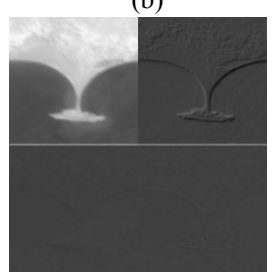

(e)

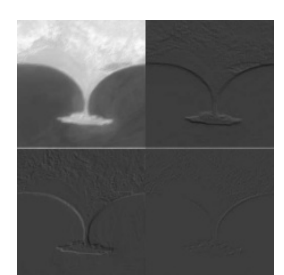

(c)

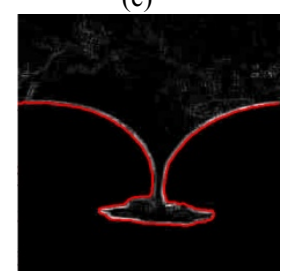

(f)
Fig. 5. Shoreline extracted directly from the SPOT-5 satellite image. (a) is original image; (b)-(e) are the images processed via non-separable wavelet filters with different parameters; (f) is the extracted shoreline which is plotted by the red curve.

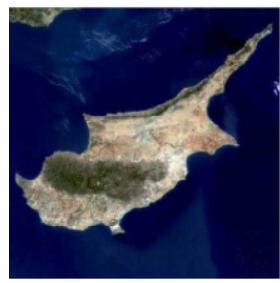

(a)

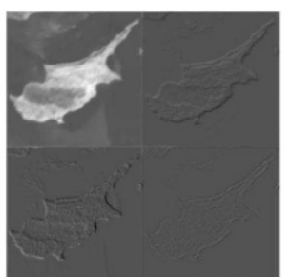

(b)



(c)

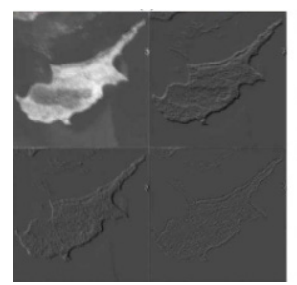

(d)

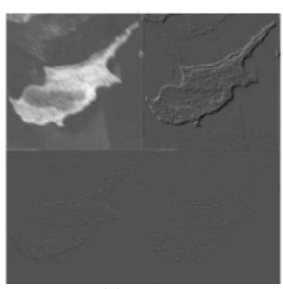

(e)

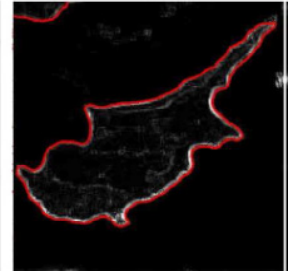

(f)
Fig. 6. Shoreline extracted directly from the Landsat-7 satellite imagery. (a) is original image; (b)-(e) are the images processed via non-separable wavelet filters with different parameters; (f) is the extracted shoreline which is plotted by the red curve.

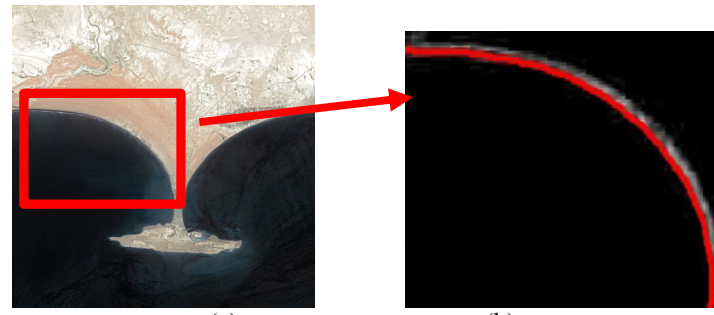

(b)

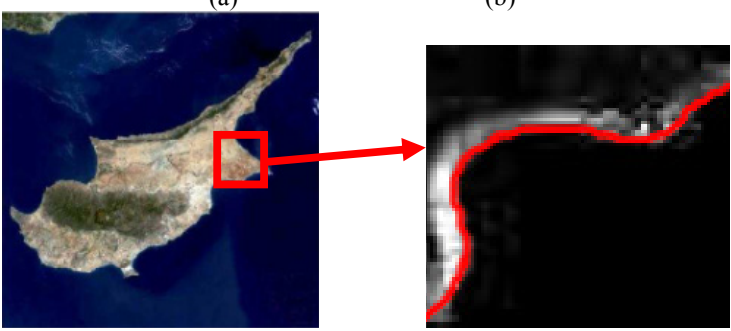

(c)
Fig. 7. Zoomed in shoreline details. (a) is the original image from SPOT-5 satellite and (c) is the original image from Landsat-7 satellite.

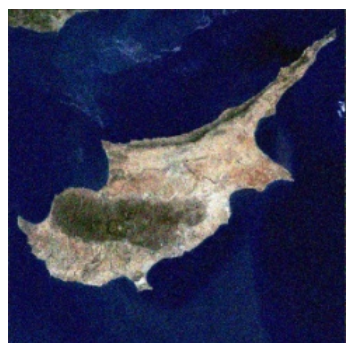

(a)



(c)

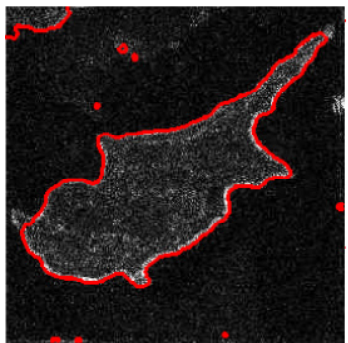

(b)

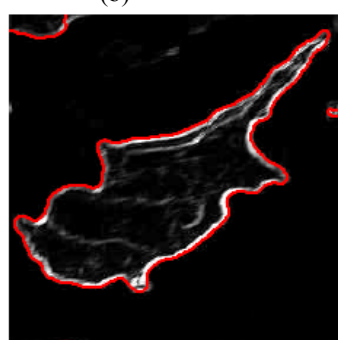

(d)
Fig. 8. Robustness of the proposed method. (a) is noised image which is contaminated by zero means Gaussian noises with variances 0.02 ; (c) is the blurred image; (b) and (d) are the extracted shorelines.

In Fig. 4 and Fig. 6, we demonstrate the final shorelines extracted directly from different satellite imagery. Fig. 4(a) is the original image from CBERS-2 satellte, Fig. 4(b) to Fig. 4(e) are the images processed via non-separable wavelet filters with different parameters, and Fig. 4(f) is the extracted shoreline which is plotted by the red curve. The same is to Fig. 5 and Fig. 6.

In Fig. 8, we have testified the robustness of our methodology in two circumstances: (1) images contaminated with Gaussian noises; and (2) images blurred by motion. According to the results, our methodology can not only robust to noises, but also have high robustness to blurring.

More results are shown in Fig. 9. 


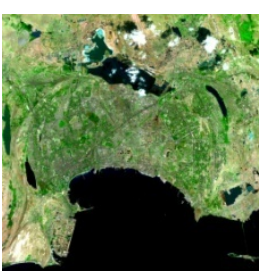

(a-1)

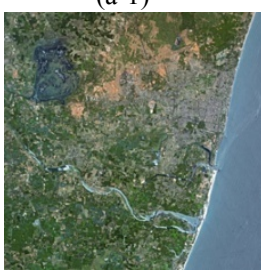

(b-1)

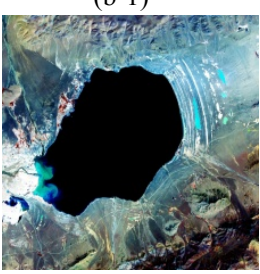

(c-1)

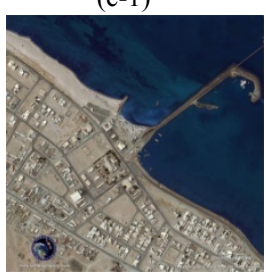

(d-1)

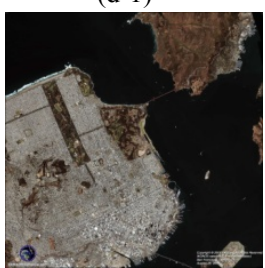

(e-1)



(f-1)



(a-2)

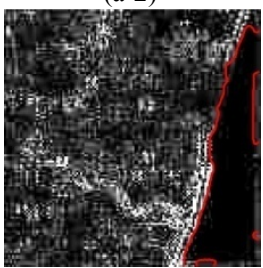

(b-2)



(c-2)

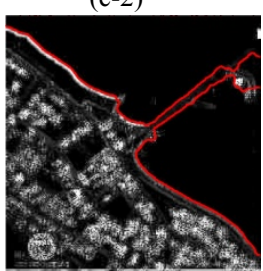

(d-2)

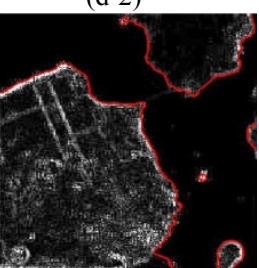

(e-2)

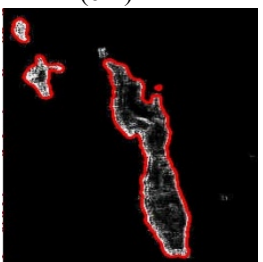

(f-2)

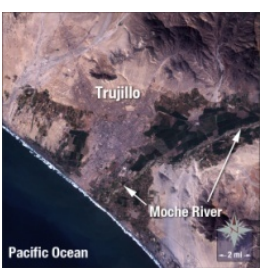

(g-1)



(h-1)



(i-1)

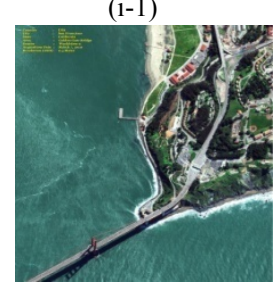

$(\mathrm{j}-1)$

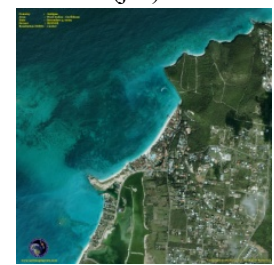

(k-1)



(1-1)

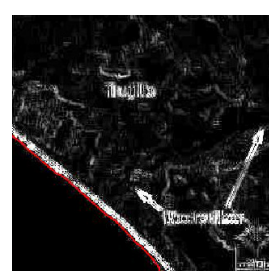

(g-2)

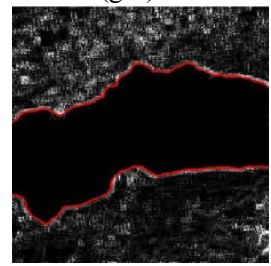

(h-2)

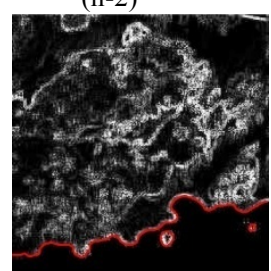

(i-2)



$(\mathrm{j}-2)$

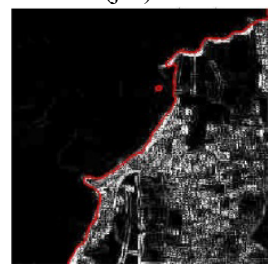

(k-2)



(1-2)

Fig. 9. Results of our algorithm. (a-1),(b-1),(c-1),(d-1),(e-1),(f-1) are original images from Landsat-7, SPOT-5, Aster, WorldView-2, IKONOS and Quick-Bird, respectively; (a-2),(b-2),(c-2),(d-2),(e-2),(f-2) are extracted shorelines corresponding to each original image. The same is to (g-1),(h-1),(i-1),(j-1),(k-1),(1-1) and (g-2),(h-2),(i-2),(j-2),(k-2),(l-2)

\section{CONCLUSION}

In this paper, we have proposed a simple but powerful algorithm for shoreline detection and extraction on single satellite image. Our proposed algorithm can not only easily detect and extract shoreline from single satellite image, but is also robust to noises and blurring.

In our experiments, the validating performance of our proposed algorithm is presented. It was shown that with non-separable wavelet, the singularities of shoreline can be detected effectively. And the amendment procedure based on distance regularized level set evolution (DRLSE) can accurately extract the shoreline according to the sub-images obtained from the first step. Additional research on robustness is also presented. It was demonstrated that our proposed algorithm could robust to not only noises but also blurring.

In the future, the algorithm described in this paper could be improved and extended works would be done in the following areas. Firstly, constructing a self-adaptive non-separable wavelet to better detect the singularities in single image; Secondly, modifying the distance regularized level set evolution (DRLSE) or adopting other creative methods to more accurately extract the shoreline.

\section{REFERENCES}

[1] H. Liu, "Automated extraction of coastline from satellite imagery by integrating Canny edge detection and locally adaptive thresholding 
methods," International Journal of Remote Sensing, vol. 25, pp. 937-958. March 2004.

[2] T. Kuleli, A. Guneroglu, F. Karsli, and M. Dihkan, "Automatic detection of shoreline change on coastal Ramsar wetlands of Turkey," Ocean Engineering, vol. 38, pp. 1141-1149, July 2011.

[3] D. T. Pugh, Changing sea levels: Effects of tides, weather and climate. Cambridge University Press, 2004, pp.78-128.

[4] S. P. Leatherman, "Historical and projected shoreline mapping," in Proc. 3rd Sympo. Coastal and Ocean Management, San Diego, 1983, pp. 2902- 2910.

[5] J. S. Lee and I. Jurkevich, "Coastal detection and tracing in SAR images," IEEE Trans. Geoscience and Remote Sensing, vol.28, pp.662 -668 , July 1990.

[6] E. H Boak and I. L. Tunner, "Shoreline definition and detection: A review," Journal of Coastal Research, vol. 24, pp. 688-703, 2005.

[7] K. W. Hsee, "Tidal datums and tide coordination," Journal of Coastal Research, Special Issue.vol.38, pp.33-43.2003.

[8] Z. Norcross, M, Fletcher and M. Merrifield, "Annual and interannual changes on a reef-fringed pocket beach: Kailua Bay, Hawaii," Marine Geology, vol.190, pp.553-580.November 2002.

[9] A. P. Cracknell, "Remote sensing techniques in estuaries and coastal zones-an update," International Journal of Remote Sensing, vol.19, 3, pp.485-496.

[10] C. M. Li, Chenyang Xu et.al., "Distance regularized level set evolution and its application to image segmentation," IEEE Trans. Image Processing, vol.19, pp.3243-3254, September 2010.

[11] E. Brannock and M. Weeks, "A synopsis of recent work in edge detection using the DWT," in Proc. IEEE Southeastcon, 2008, pp. $515-520$.

[12] Y. P. Guan, "Automatic extraction of lips based on multi-scale wavele tedge detection," Comput. Vis., vol. 2, pp.23-33, Mar. 2008.

[13] F. Pellegrino, W. Vanzella, and V. Torre, "Edge detection revisited," IEEE Trans. Syst., Man, Cybern., vol. 34, pp. 1500-1518, July 2004.

[14] C. M. Li, Chenyang Xu, et. al., "Distance regularized level set evolution and its application to image segmentation," IEEE Trans. Image Processing, vol.19, pp.3243-3254, Sept. 2010.

[15] M. Sussman, P. Smereka, and S. Osher, "A level set approach for computingsolutions to incompressible two-phase flow," J. Comput. Phys, vol. 114, pp. 146-159, Sept. 1994.

[16] M. Sussman and E. Fatemi, "An efficient, interface-preserving level set redistancing algorithm and its application to interfacial incompressible fluid flow," SIAM J. Sci. Comput, vol. 20, pp. 1165-119, July 1999.

[17] D. Peng, B. Merriman, S. Osher, H. Zhao, and M. Kang, "A PDE based fast local level set method," J. Comput. Phys., vol. 155, pp. 410-438, Nov. 1999.

[18] X. You, D. Zhang, and Q. Chen, "Face representation by using non-tensor product wavelets," in Proc.18th International Conference on Pattern Recognition, HongKong, 2006, pp.503-506.

[19] X. G. You, L. Du, "A blind watermarking scheme using new non-tensor product wavelet filter banks," IEEE Transactions on Image Processing, vol.19, pp.3271-3284, Dec. 2010.

[20] J. Huang and X. G. You, "Rotation invariant iris feature extraction using Gaussian Markov random fields with non-separable wavelet," Neurocomputing, vol 3. pp. 883-894, Mar. 2010.

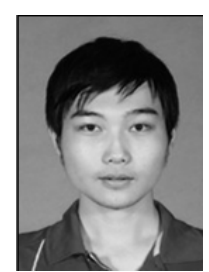

Shujian Yu is a senior student in the Department of Electronics and Information Engineering, Huazhong University of Science and Technology, Wuhan, China.

His current research interests include wavelets and their applications, image processing, especially saliency detection, image stitching and Hyper-spectral image processing.



Yi Mou received the B.S. degree in Wuhan University of Science and Technology, Wuhan, China, and M.S. degree in Wuhan Polytechnic University, Wuhan, China in 2004, 2009, respectively.

His current research interests include wavelets and their applications, image processing, computer vision, especially Multi-spectral signal processing and Hyper-spectral image processing.

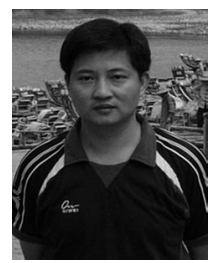

Duanquan Xu received the B.S. degree in Electronics and Information Engineering from Wuhan University, Wuhan, China, M.S. degree in China Ship Research and Development Academy, Beijing, China, and Ph.D. degree in Electronics and Information Engineering, Huazhong University of Science and Technology, Wuhan, China in 1991, 2002, and 2008, respectively.

His current research interests include Biomedical image processing, pattern recognition and digital watermarking.

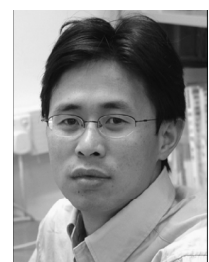

Xinge You received the B.S. and M.S. degrees in mathematics from the University of Hubei, Wuhan, China, and the Ph.D. degree in computer science from the Hong Kong Baptist University, Hong Kong, in 1990, 2000, and 2004, respectively.

$\mathrm{He}$ is presently a Professor in the Department of Electronics and Information Engineering, Huazhong University of Science and Technology, Wuhan, and an Adjunct Professor in the School of Computer Science, Chongqing University, Chongqing, China. His current research interests include wavelets and their applications, signal and image processing, pattern recognition, and computer vision.

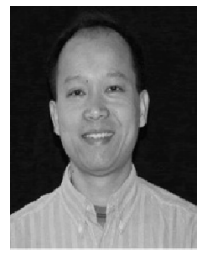

Long Zhou received Ph.D. degree from Huazhong University of Science and Technology, Wuhan, China, in 1998.

$\mathrm{He}$ is presently a Professor in Electrical and Electronic Engineering, Wuhan Polytechnic University, Wuhan, China. His current research interests include the theory of neural networks and intelligent image processing and its application.

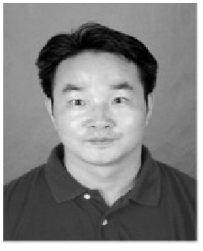

Wu Zeng received the B.S. and M.S. degrees from the University of Hubei, Wuhan, China, in 1992, 2002, respectively.

$\mathrm{He}$ is presently an Associate Professor in Electrical and Electronic Engineering, Wuhan Polytechnic University, Wuhan, China. His current research interests include artificial intelligence, information theory and its application. 